\title{
Evaluation of maternal near miss cases in tertiary care centre
}

\author{
Vandana Ojha, Amrita Chaurasia, Shalini Singh, Nidhi Sachan, Shadma Siddiqui \\ Correspondence: Dr Amrita Chaurasia, Professor \& Head, Department of Obstetrics \& \\ Gynaecology , MLN Medical College, Prayagraj, UP, India; Email - \\ dr.amrita.chaurasia@gmail.com \\ Distributed under Attribution - NonCommercial - Share Alike 4.0 International (CC BY-NC-SA 4.0)
}

\begin{abstract}
Objective: A study to analyze maternal near miss obstetrics event and maternal mortality in a tertiary care centre. Methods: This was a retrospective analysis of data, from January 2015 to December 2017. For each eligible patient, medical records were reviewed. Their socio-demographic features, mode of delivery, diagnosis on admission, surgical intervention, ICU admission, duration of hospital stay and outcome were collected and analyzed. Results: There were 159 maternal near miss cases and 83 maternal deaths. Maternal near miss to mortality ratio was 1.92 . During the study maternal near miss incidence (MNM ratio) was found to be 47.73 per 1000 live birth. Mortality index in our study was 34.29. There was more than one delay in most of the patients. Delays were primarily in seeking health care and delayed referral. Haemorrhage, hypertensive disorders and rupture uterus are most common cause of maternal near miss in our study. Conclusion: Investigating the maternal near miss cases are important tool to get the information about the causes, identify the gaps and corrective measures to be taken in the health care system.
\end{abstract}

Keywords: Maternal near miss, maternal death, MNMR.

Complications during pregnancy and child birth can occur at any point of time ${ }^{1}$. In any of the medical setup, pregnant women who develop severe acute morbidity share more or less same pathological and circumstantial factors. While some of these women die, a proportion of luckier ones narrowly escapes death and is termed as "Near Miss Cases". By evaluating these cases with severe maternal morbidities and mortalities (both "near-miss" cases and maternal deaths), much can be learnt about the processes in place to deal with maternal morbidities. ${ }^{2}$ Maternal near-miss cases are defined as "a woman who nearly died but survived a complication that occurred during pregnancy, childbirth or within 42 days of termination of pregnancy" ${ }^{2}$. In practical terms, women are considered near-miss cases when they survive lifethreatening conditions (i.e. organ dysfunction) ${ }^{2}$. Maternal near-miss cases can directly inform on problems and obstacles that had to be overcome during the process of health care facility improvement and thus their audits have been considered as useful approaches to improve maternal health care ${ }^{3}$. Additionally investigating near miss events are advantageous since we can learn from the women themselves since they survived the event and are available for interview about the care they received. All near misses should be interpreted as free lessons and opportunities to improve the quality of service provision. ${ }^{1}$

Methods

This retrospective observational study was carried out

Received: $29^{\text {th }}$ April 2019. Accepted: $7^{\text {th }}$ June 2019.

Ojha V, Chaurasia A, Singh S, Sachan N, Siddiqui S. Evaluation of maternal near miss cases in tertiary care centre.

The New Indian Journal of OBGYN. 2019; 6(1): 45-8. 
in the Department of Obstetrics and Gynaecology, MLN Medical College, Prayagraj from January 2015 to December 2017. Due to limitation of resources, purposive sampling method was used to collect samples. Sample collection was done from medical records. Medical level ${ }^{5}$. Statistical calculations were done by applying Innova test and calculating $\mathrm{P}$ value.

\section{Results}

During the study period, there were 3744 total deliveries including both vaginal and caesarian births, out records were reviewed, their sociodemographic features, mode of delivery, diagnosis on admission, Table 1: Distribution based on demographic variables $(\mathrm{N}=159)$. surgical intervention, ICU admission, duration of hospital stay and ultimate outcome were collected. Women were categorized as per laboratory, clinical and management based criteria laid down by WHO 2009. The collected data were recorded and analyzed.

Inclusion Criteria: Critically ill Table 1: Distribution based on demographic variables (N=159).

\begin{tabular}{lll}
$\begin{array}{l}\text { Maternal Near } \\
\text { Miss cases } \\
\text { Number }(\%)\end{array}$ & $\begin{array}{l}\text { Total deliveries } \\
(\mathbf{N}=3744)\end{array}$ & P-value \\
\hline $39(24.52 \%)$ & $1071(28.61 \%)$ & 0.264318 \\
$120(75.76 \%)$ & $2673(71.39 \%)$ & \\
$112(71.00 \%)$ & $3115(83.20 \%)$ & 0.00001 \\
$42(29.00 \%)$ & $610(16.28 \%)$ & \\
$119(74.84 \%)$ & $2283(61 \%)$ & 0.000438 \\
$40(25.15 \%)$ & $1460(39 \%)$ & \\
$50(31.44 \%)$ & $779(20.80 \%)$ & 0.00171 \\
$109(68.55 \%)$ & $2927(78.19 \%)$ & \\
$61(38.36 \%)$ & $1066(28.48 \%)$ & 0.007068 \\
$98(61.63 \%)$ & $2677(71.51 \%)$ & \\
\hline
\end{tabular}

pregnant, labouring, postpartum and postabortal women admitted to department Obstetrics and Gynaecology MLN Medical College, Prayagraj, fulfilling the WHO criteria of near miss.

Exclusion Criteria: Women that developed life threatening conditions unrelated to pregnancy i.e. not during pregnancy or 42 days after termination of pregnancy were excluded.

The following indices were calculated -1) Maternal near-miss (MNM) Case - referring to women who nearly died but survived a complication during pregnancy, childbirth or within 42 days of termination of pregnancy. 2) Maternal death (MD ) - referring to the death of a woman while in pregnancy or within 42 days of termination of pregnancy or its management, but not from accidental or incidental cause. 3) Live birth (LB) referring to the birth of offsprings who breath or showed evidence of life. 4) MNM ratio (MNMR) - referring to the number of maternal near - miss cases per 1000 live birth. 5) Maternal near-miss mortality ratio (MNM: MD) - referring to the ratio between maternal near miss cases and maternal deaths. 6) Mortality index - referring to the number of maternal deaths divided by the number of woman with life threatening conditions expressed as a percentage $[\mathrm{MI}=\mathrm{MD} /(\mathrm{MNM}+\mathrm{MD})]^{2}{ }^{2}$ 7) Delays - First delay: delay in deciding to seek care; Second delay: delay in reaching an appropriate health facility; Third delay: delay in receiving quality obstetric care at health facility

of which 3331 were live births, 159 were near-miss cases
as per WHO set of severity markers for acute maternal morbidity and 83 were maternal deaths. MNM ratio (MNMR), Maternal near miss to mortality ratio [MNM: MD] and Mortality index are 47.73 per 1000 live birth, 1.92 and 34.29 respectively. On analyzing the age statistics, although statistically not significant, $75.76 \%$ $(n=120)$ cases belong to age group of $>20$ years. On analyzing educational statistics $71 \%$ of cases were illiterate $(\mathrm{p}=.00001)$. Maternal near miss belong to rural area $74.84 \%(n=119)$ was significant $(p=.00043)$. Number

Table 2: Distribution of patients based on types of delays affecting maternal morbidities and moralities $(\mathrm{N}=159)$

\begin{tabular}{ll}
\hline Delays & Number (\%) \\
\hline First delay & $54(33.96 \%)$ \\
Second delay & $16(10.06 \%)$ \\
Third delay & $7(4.4 \%)$ \\
More than one delays & $74(46.54 \%)$ \\
Delays unidentified & $8(5.03 \%)$ \\
\hline
\end{tabular}

of unbooked and multigravidas cases were significant (Table 1). Table 2 - shows types of delay in near miss cases. First delay (delay in women seeking help) delay identified in $33.96 \%$ of cases. Second delay (logistic) identified in $10.06 \%$ of cases. Third delay (facility level) delay identified in $4.4 \%$ of cases. More than one delay identified in $46.54 \%$ of cases. Table 3 shows that hypertensive disorders (29.55\%), hemorrhage (20.75\%), rupture uterus $(20.12 \%)$ are the most common causes for near miss cases. Table 4 shows intervention needed in the 
Table 3: Distribution of patient based on obstetrical complications $(\mathrm{N}=159)$

\begin{tabular}{lc}
\hline Obstetrical complications & Number (\%) \\
\hline Hypertensive disorder & $47(29.55 \%)$ \\
APH AND PPH & $33(20.75 \%)$ \\
Rupture uterus & $32(20.12 \%)$ \\
Rupture ectopic pregnancy & $18(11.32 \%)$ \\
Uterine inversion & $4(2.51 \%)$ \\
Septic abortion & $8(5.03 \%)$ \\
Puerperal sepsis & $4(2.51 \%)$ \\
Liver dysfunction & $7(4.40 \%)$ \\
Cardiac dysfunction & $4(2.51 \%)$ \\
Respiratory dysfunction & $2(1.25 \%)$ \\
\hline management. ICU admission was & needed in $33.96 \%$ \\
cases and cardiopulmonary & resuscitation was done \\
8.17\% of cases. Cardiotonics & were given in $47.16 \%$ \\
Table 4: Intervention needed in the management of \\
maternal near miss cases (N=159) & \\
\hline Intervention needed & Number (\%) \\
\hline ICU admissions & $54(33.96 \%)$ \\
Cardiopulmonary resuscitation & $13(8.17 \%)$ \\
Mechanical ventilation & $46(28.90 \%)$ \\
Use of cardio tonic & $75(47.16 \%)$ \\
Massive blood/blood product & \\
transfusion & $29(18.23 \%)$ \\
Renal or peritoneal dialysis & $4(2.51 \%)$ \\
Laparotomy & $65(40.88 \%)$ \\
\hline
\end{tabular}

cases. Laparotomy was done in $40.88 \%$ of cases. (Many near miss patients needed more than one intervention during their management).

\section{Discussion}

Most of the maternal near miss cases result from a chain of events that not only includes medical factors; many social, cultural factors too have significant impact. Overall, $80 \%$ of MNM cases are preventable. Majority of maternal near-miss cases were from rural background and were unbooked, despite being experienced of previous births referred from other health facilities. Multipara patients were more common. Most of the unbooked patients belong to low socioeconomic status and illiterate. The poor economic and education status may make it difficult for women to seek care. Delays were primarily in seeking health care and delayed referral. The first two delays are related directly to factors in family and community, whereas the third delay is connected with facility and quality of care. In developing countries, about $75 \%$ of women with severe obstetric morbidity are in critical condition upon arrival underscoring the significance of first two delays . ${ }^{4-7}$

Maternal near miss incidence ratio (MNM ratio) in our study is somewhat higher than various Indian studies, like study done by Sarma et al ${ }^{8}$ in Assam had ratio of 42.09 per 1000 live births while study done by Kalra et al ${ }^{9}$ in Western Rajasthan had a ratio of 4.18 per 1000 live birth. MNM ratio, gives an estimation of the amount of care and resources that would be needed in the facility. Since our institution is a tertiary centre, most of the patients are referred, and they are already in moribund condition.

Referral is the process of coordinated movement of health care seeker to reach a high-level care within a small window of time ${ }^{10}$. The goal of timely referral is to minimize or prevent the delay for transportation (called second delay), and ensure pre-hospital care while transporting a patient to the referral facility. ${ }^{11,12}$ Referral form should be properly filled and there should be communication between two facilities. Maternal near miss to mortality ratio [MNM:1MD] is comparable to study by Bansal et al ${ }^{13}$ with near miss to mortality ratio 2.05:1 which means every two women who survived life threatening WHO severity markers, one woman died. Higher ratio indicates better care. Study done in Nepal showed a ratio of $7.2: 1^{14}$, while study done in Western Europe showed a ratio of 117.22: $1^{15}$.

Mortality index in our study is comparable to study done by Singh et al ${ }^{16}$ with mortality index of 32.58 , while study done by Madhvi et al ${ }^{17}$ found the index of 8.3. The higher the index the more women with life threatening conditions die (low quality of care), whereas the lower the index the fewer women with life threatening conditions die (better quality of care).

Hemorrhage, hypertensive disorders and rupture uterus are most common cause of maternal near miss in our study. Various other Indian studies also shown that hemorrhage and eclampsia are most common cause for maternal near miss. Monitoring of component of antenatal care must be done regularly. Pregnant patients with previous LSCS must be properly counseled about the importance of proper antenatal checkups. Alarming signs should be properly explained. Patients with high blood pressure should be counseled about the regular monitoring of blood pressure, use of antihypertensive drugs, signs for imminent eclampsia should be explained in patients' 
language at all level of health care. Regular PPH and eclampsia drills should be organized at all PHC and CHC. Anemia should be treated in all patients by oral iron, intravenous iron or by blood transfusion depending on severity of anemia and gestational age. The health workers, ANMs, ASHA workers should be trained in recognizing the high risk patients and the warning symptoms and signs of pregnancy and hence early referral of these women to the nearest institutions. Contraceptive services must be strengthened.

\section{Conclusion}

Continuous review of the existing infrastructure and ongoing improvements are required as per the demand. Multipronged approach should be implemented by improved literacy, social awareness and health system impediments at the grass root level with improved tertiary level. Establishing Obstetric HDU / ICUs in department of obstetrics and gynecology of state medical colleges by NHM is such type of effort.

\section{Conflict of interest: None. Disclaimer: Nil. References}

1.Ministry of health and family welfare. Maternal Near Miss Review - Operational Guidelines. New Delhi: Maternal Health Division Ministry of Health \& Family Welfare Government of India; 2014.

2.WHO. Evaluating the quality of care for severe pregnancy complications. The WHO near-miss approach for maternal health. Geneva, Switzerland: WHO; 2011.

3.Pattinson RC, Vandecruys HI, Macdonald AP, Mantel GD. Why do women die during childbirth. 2001.

4.Gelany SE, Mansour MM, Hassan MM. The Three delays of maternal mortality in a public-sector tertiary teaching hospital: is there a paradigm shift? Gynecol Obstet Res Open J. $2015 ; 2$ : 52-6.

5.Rai NK, Dali SM. Making pregnancies safer in South-East Asia. WHO South-East Asia. Region Health Forum. 2002; 6:19-28.

6.Mustafa R, Hashmi H. Near-miss obstetrical events and maternal deaths. J Coll Physicians Surg Pak. 2009; 19:781-5.

7.Roost M, Altamirano VC, Liljestrand J, Essén B. Priorities in emergency obstetric care in Bolivia: maternal mortality and near-miss morbidity in metropolitan La Paz. BJOG. $2009 ; 116: 1210$ - 7 .
8.Sarma HKD, Sarma HK, Kalita AK. A prospective study of maternal near-miss and maternal mortality cases in FAAMCH, Barpeta; with special reference to its aetiology and management: first 4 months report. J Obstet Gynaecol Barpeta. 2015; 1(2): 100-6.

9.Kalra P, Kachhwaha CP. Obstetric near miss morbidity and maternal mortality in a Tertiary Care Centre in Western Rajasthan. Indian J Publ Health. 2014; 58(3).199-201.

10.Giovine A, Ostrowski C. Technical report on Improving Transportation and Referral for Maternal Health: Knowledge Gaps and Recommendations. Wilson Cent. 2010.

11.Raj SS, Manthri S, Sahoo PK. Emergency Referral Transport for Maternal Complication: Lessons from the Community Based Maternal Death Audits in Unnao District, Uttar Pradesh, India. Int J Health Policy Manag. 2015; 4(2): 99-106.

12.Potluri P. Emergency services in India- Counting on betterment. A transition in progress. Editorial Section. [Last assessed on 2018 Jan 25]; Asian Hospital and health care Management magazine. 2009. Available from:

http://www.asianhhm.com/healthcare_management/emergen cy_services_india.htm.

13.Bansal M, Lagoo J, Pujari K. Study of near miss cases in obstetrics and maternal mortality in Bastar, Chhattisgarh, India. Int J Reprod Contracep Obstet Gynecol. 2017 Feb 23; 5(3): 620-3.

14. Shrestha NS, Saha R, Karki C. Near miss maternal morbidity and maternal mortality at Kathmandu Medical College Teaching Hospital. Kathmandu University Medical Journal. 2010; 8(30): 222-6.

15.van Roosmalen J, Zwart J. Severe acute maternal morbidity in high-income countries. Best Practice and Research: Clinical Obstetrics and Gynaecology. 2009; 23(3): 297-304.

16.Abha S, Chandrashekhar S, Sonal D. Maternal near miss: a valuable contribution in maternal care. J Obstet Gynecol India. 2016 Oct 1; 66(1): 217-22.

17.Madhavi Nacharaju SS, Kaul R, Reddy P. Maternal near miss: an experience in rural medical college. J Evol Med Dental Sci. 2014; 3(56): 12761-7.

\footnotetext{
Vandana Ojha ${ }^{1}$, Amrita Chaurasia ${ }^{2}$, Shalini Singh ${ }^{3}$, Nidhi Sachan ${ }^{4}$, Shadma Siddiqui ${ }^{5}$

${ }^{1,3,4}$ Lecturer; ${ }^{2}$ Professor \& Head; Junior Resident , Department of Obstetrics \& Gynaecology, MLN Medical College ,Prayagraj, UP, India.
} 